\title{
The indicative function of Twist2 and E-cadherin in HPV oncogene-induced epithelial-mesenchymal transition of cervical cancer cells
}

\author{
YUAN LIU ${ }^{1 *}$, WENYAN QIAN ${ }^{2 *}$, JIAWEN ZHANG $^{3}, \mathrm{YU} \mathrm{DONG}^{1}, \mathrm{CAN} \mathrm{SHI}^{1}, \mathrm{ZHIQIANG} \mathrm{LIU}^{4}$ and SUFANG WU ${ }^{1}$ \\ ${ }^{1}$ Department of Obstetrics and Gynecology, Shanghai First People's Hospital, Shanghai Jiaotong University, Shanghai; \\ ${ }^{2}$ Department of Gynecology and Obstetrics, Kunshan Hospital of Traditional Chinese Medicine Affiliated to \\ Nanjing University of Chinese Medicine, Jiangsu; ${ }^{3}$ Department of Obstetrics and Gynecology, \\ Shanghai Tenth People's Hospital, Shanghai Tongji University, Shanghai, P.R. China; ${ }^{4}$ Division of Cancer Medicine, \\ Department of Lymphoma and Myeloma, Center for Cancer Immunology Research, \\ The University of Texas MD Anderson Cancer Center, Houston, TX, USA
}

Received July 29, 2014; Accepted October 17, 2014

DOI: $10.3892 /$ or.2014.3620

\begin{abstract}
High-risk human papillomavirus (HR-HPV) infections are among the most important factors for cervical carcinogenesis. However, whether patients infected with HR-HPV eventually develop a malignant tumor, largely depends on epithelial-mesenchymal transition (EMT), which plays an extraordinary role in the process of carcinogenesis and metastasis. Therefore, we evaluated the protein levels of EMT-related genes in normal cervical squamous epithelium, cervical intraepithelial neoplasia (CIN), and cervical squamous cell carcinoma (SCC) by tissue microarray and immunohistochemical staining. By comparing the expression of EMT-related proteins in 31 cases of cervical tumors and tumor adjacent tissues and exploring the relationship between HPV16 oncogenes and EMT in vitro, we found that Twist2 protein levels were significantly higher in CIN and cervical cancer than in normal cervical squamous epithelial samples $(\mathrm{p}<0.01$ and $\mathrm{p}<0.001$, respectively). This finding corresponded with the decreased expression of E-cadherin in cervical cancer. The difference in the expression of Twist 2 and E-cadherin between 31 cases of cervical tumors and tumor adjacent tissues was statistically significant $(\mathrm{p}<0.01)$. HPV16 oncogenes were able to induce morphological alterations in the SiHa cell line, upregulate the expression of Twist 2 and vimentin, downregulate E-cadherin in vitro, and exert an effect on invasion. Thus, joint detection of Twist 2 and E-cadherin expression can help
\end{abstract}

Correspondence to: Dr Sufang Wu, Department of Obstetrics and Gynecology, Shanghai First People's Hospital of Shanghai Jiaotong University, Shanghai 200080, P.R. China

E-mail: wsf_sfph@163.com

*Contributed equally

Key words: human papillomavirus 16, epithelial-mesenchymal transition, Twist2, E-cadherin, cervical cancer evaluate and provide greater insight into cervical carcinogenesis and progression.

\section{Introduction}

Cervical cancer is the second leading cause of death among women worldwide. High-risk human papillomavirus (HR-HPV) infection is one of the major risk factors for cervical cancer; in particular, type 16 and 18 HR-HPV are responsible for more than $68 \%$ of cervical cancer cases (1). It has been well demonstrated that HPV persistence and the chromosomal rearrangements of two early genes, E6 and E7, are critical occurrences in HPV-associated cancers. The oncogenes E6 and E7 can disturb the cell cycle by inhibiting tumor-suppressor genes, namely p53 and retinoblastoma tumor-suppressor protein (pRB), respectively (2-5). E6 binds to the central region of p53 and induces its degradation via the ubiquitin pathway (6), while E7 protein binds and induces the degradation of the growth suppressive form of $\mathrm{pRB}$ and promotes cell cycle progression by destabilizing the $\mathrm{pRb}-\mathrm{E} 2 \mathrm{~F}$ complex. This action of E7 causes persistent activation of E2F transcription factors. All of the mechanisms mentioned above help HPV evade host immuno-surveillance, allow viral persistence, and alter cell cycle and apoptosis control thereby facilitating the accumulation of DNA damage or mutations (6). Although cervical cancer is intimately related to HPV infection, not all patients infected with HPV eventually develop cervical cancer. HPV has been reported to be a necessary but not sufficient cause of cervical cancer. Therefore, the question still remains as to what is the critical step in carcinogenesis.

The epithelial-mesenchymal transition has been shown to be involved in carcinogenesis, cancer progression and cancer metastasis (7-11). Downregulation of E-cadherin has been regarded as the vital marker for the loss of connection between cells in epithelial-mesenchymal transition (EMT) events (10-12). E-cadherin transcription and protein levels can be downregulated by EMT-activating transcription factors, 
such as the Twist family of bHLH factors (Twist1 and Twist2) which act as transcription repressors to regulate metastasis. At the same time, Twist2 promotes EMT through downregulation of E-cadherin in subsets of breast cancer (12-14). Moreover, highly expressed Twist 2 protein is mainly found in cervical intraepithelial neoplasias and cervical squamous cell carcinoma tissues (13-15).

Recently, studies have confirmed that HPV16 can induce the EMT-like process. For example, HPV16 E6/E7-immortalized human gingival keratinocytes cells demonstrated EMT-like properties (16). Forced expression of HPV16 E7 can mediate EMT in the process of cervical carcinogenesis $(17,18)$. Caberg et al (19) discovered that silencing of the E7 gene could upregulate the expression of E-cadherin. Mühlen et al (20) demonstrated that HPV16 E2 activates targets such as ERK1/2, AP-1 and MMP-9, suggesting that it plays a role in cancer carcinogenesis and metastasis. It remains to be determined whether HPV16 E2/E6/E7 induce EMT in cervical cancer cells.

In the present study, we used tissue microarray and immunohistochemical staining to evaluate the protein levels of E-cadherin and Twist 2 and found that Twist 2 expression was gradually increased during the progression from normal cervical squamous epithelium to cervical intraepithelial neoplasia (CIN) and cervical squamous cell carcinoma. At the same time, E-cadherin expression was downregulated. We found significant differences in the expression of E-cadherin and Twist 2 between the 31 cases of cervical tumor tissues and tumor adjacent tissues. In addition, we explored the impact of HPV16 oncogenes on the EMT process of cervical cancer cells. Thus, our results provide novel insight into cervical carcinogenesis and a new evaluation for cervical cancer progression.

\section{Materials and methods}

Cell culture and transfection. The human cervical carcinoma SiHa cell line (HPV16-positive) purchased from ATCC, was cultured in Dulbecco's modified Eagle's medium (DMEM) F12 1:1 medium containing 10\% fetal bovine serum (FBS) (both from Gibco, Gaithersburg, MD, USA) and a $100 \mu \mathrm{g} / \mathrm{ml}$ penicillin/streptomycin antibiotic mixture at $37^{\circ} \mathrm{C}$ with $5 \% \mathrm{CO}_{2}$.

Before transfection, the $\mathrm{SiHa}$ cells were cultured on 6-ml dishes in $5 \mathrm{ml}$ of growth medium. The p-CAG-MYCHPV16 E2 plasmid, p-CAG-MYC-HPV16 E6 plasmid, p-CAG-MYC-HPV16 E7 plasmid and the empty vector were donated by Dr Sufang Wu (Shanghai First People's Hospital, Shanghai Jiaotong University). After serum starvation for $24 \mathrm{~h}$, the SiHa cells were transfected with the plasmids using Lipofectamine ${ }^{\mathrm{TM}} 2000$ (Invitrogen) according to the manufacturers' protocol.

$R T$-PCR. Total RNA was extracted with TRIzol (Invitrogen). For microarray hybridization, cDNA was produced from $2.5 \mu \mathrm{g}$ of total RNA according to the Takara manuscript. Thirty-four PCR cycles were used for HPV16 E2, E6, E7, E-cadherin, vimentin, Twist 2 and 28 PCR cycles for GAPDH. The objective gene was amplified using appropriate primers (Table I).

Western blot analysis. After the harvested cells were lysed and the supernatant was collected, then, $60 \mu \mathrm{g}$ of protein was
Table I. Primers used in this study.

\begin{tabular}{ll}
\hline Primer & \multicolumn{1}{c}{ Sequence (5' to 3') } \\
\hline E2 & F: TCTGTGTTTAGCAGCAACGAA \\
& R: TAATCCGTCCTTTGTGTAGC \\
E6 & F: CGACCCAGAAAGTTACCACAGT \\
& R: AATCCCGAAAAGCAAAGTCATA \\
E7 & F: GAGGAGGAAGATGAAATAGATGG \\
& R: AACCGAAGCGTAGAGTCACAC \\
E-cadherin & F: TTGCTACTGGAACAGGGACAC \\
Vimentin & R: CCCGTGTGTTAGTTCTGCTGT \\
& F: AGATGGCCCTTGACATTGAG \\
Twist2 & R: CCAGAGGGAGTGAATCCAGA \\
& F: ACAAGCTGAGCAAGATCCAGAC \\
GAPDH & R: GCTGGTCATCTTATTGTCCATCT \\
& F: AGAAGGCTGGGGCTCATTTG \\
& R: AGGGGCCATCCACAGTCTTC
\end{tabular}

F, forward; R, reverse.

loaded onto an SDS-PAGE and transferred to polyvinylidene fluoride (PVDF) membranes. The membranes were blocked with $5 \%$ skimmed milk for $1.5 \mathrm{~h}$ and incubated overnight with one of the following primary antibodies: anti-E-cadherin (diluted at 1:500), anti-vimentin (diluted at 1:200), anti-Twist2 (diluted at 1:500) (all from Abcam) and anti-GAPDH (diluted at 1:1,000; Cell Signaling Technology) rabbit polyclonal antibody, anti-HPV16 E2 (diluted at 1:500), anti-HPV16 E6 (diluted at 1:500) (both from Abcam) and anti-HPV16 E7 (diluted at 1:100; Beijing, Biosynthesis Biotechnology Co.) rabbit polyclonal antibody. The reactions were then incubated with the appropriate secondary antibody $(1: 5,000)$.

Immunofluorescence. A total of $2.5 \times 10^{4}$ cells were seeded on a glass cover in a 24 -well microtiter plate for each time period. Thirty-six hours after introduction of the plasmids, the cells were fixed with $4 \%$ paraformaldehyde in PBS, permeabilized by incubation with PBS $+0.01 \%$ Triton X-100 (Sigma) for $5 \mathrm{~min}$, and stained with anti-Twist 2 antibody (ab66031; Abcam) in PBS $+5 \%$ sheep serum for $1 \mathrm{~h}$. The secondary antibody was Alexa Fluor 488-conjugated anti-rabbit antibody (\#A11001; Invitrogen) in PBS + 5\% sheep serum. The nuclei were counterstained with 4',6-diamidino-2-phenylindole (DAPI). Photographs were captured using a Zeiss Axiophot fluorescence microscope equipped with Axiovision software version 4.6 (Carl Zeiss).

Immunohistochemistry of the tissue microarray. Cervical intraepithelial neoplasia tissue microarray (CIN481) and cervical squamous cancer tissue microarray (CXC962 and Utr03-003) were purchased from Shanghai Zuocheng Biology Co. containing normal cervical squamous epithelial tissue (6 cases), CIN I-II (19 cases), CIN III (11 cases), and cervical squamous cell carcinoma tissue ( 70 cases). There were 31 pairs of cervical tumor tissues and tumor adjacent tissues. The tumor 
Table II. Association between the clinicopathological features and the expression of Twist2 in cervical disease tissues.

\begin{tabular}{|c|c|c|c|c|c|}
\hline & \multicolumn{2}{|c|}{ Twist 2 expression in cytoplasm } & \multirow[b]{2}{*}{ Positive rates } & \multirow[b]{2}{*}{$\chi^{2}$} & \multirow[b]{2}{*}{ P-value } \\
\hline & - & + to +++ & & & \\
\hline Tissue type & & & & 36.938 & 0.000 \\
\hline Normal & 6 & 0 & $0.0 \%$ & & \\
\hline CIN I-II & 8 & 11 & $57.9 \%$ & & \\
\hline CIN III & 3 & 8 & $72.7 \%$ & & \\
\hline SCC & 3 & 67 & $95.5 \%$ & & \\
\hline Total $(n=106)$ & 20 & 86 & & & \\
\hline Age (years) & & & & 0.220 & 0.801 \\
\hline$\leq 45$ & 13 & 51 & $79.7 \%$ & & \\
\hline$>45$ & 7 & 35 & $83.3 \%$ & & \\
\hline Total $(n=106)$ & 20 & 86 & & & \\
\hline Differentiation grade of tumor cells & & & & 2.178 & 0.588 \\
\hline High & 0 & 2 & $100.0 \%$ & & \\
\hline Moderate & 1 & 42 & $97.7 \%$ & & \\
\hline Low & 2 & 23 & $92.0 \%$ & & \\
\hline Total $(n=70)$ & 3 & 67 & & & \\
\hline Lymph node metastasis & & & & 0.264 & 0.521 \\
\hline Negative & 2 & 53 & $96.4 \%$ & & \\
\hline Positive & 1 & 14 & $93.3 \%$ & & \\
\hline Total $(n=70)$ & 3 & 67 & & & \\
\hline
\end{tabular}

CIN, cervical intraepithelial neoplasia; SCC, squamous cell carcinoma.

tissues were also included in the 70 cases of cervical squamous cell carcinoma tissues but the tumor adjacent tissues were not included in the 6 cases of normal cervical squamous epithelial tissue since they encompassed not only normal cervical tissues but also CIN tissues that we did not distinguish.

Immunohistochemistry was performed according to the manufacturer's instructions. Rabbit anti-E-cadherin Ab 1:100 and anti-Twist $2 \mathrm{Ab}$ 1:100 were used for staining.

Invasion assay. To perform the invasion assays, the cells were plated ( $10^{5}$ cells/chamber) in BD BioCoat Matrigel invasion chambers (BD Biosciences). In the upper chamber, the medium was supplemented with $2 \%$ heat-inactivated FBS. In the lower chamber, $20 \%$ FBS was used as a chemoattractant. After $24 \mathrm{~h}$, the medium was removed and the chambers were washed twice with PBS. Non-invading cells were removed from the upper surface of the membrane by scrubbing with a cotton-tipped swab, and invading cells were fixed with $3.7 \%$ formaldehyde in PBS for 2 min, washed with PBS twice, permeabilized with methanol for $20 \mathrm{~min}$, washed twice with PBS, stained with $0.05 \%$ crystal violet for $15 \mathrm{~min}$, and washed twice with PBS. For each chamber, five fields for each chamber were photographed using a digital camera mounted on an inverted microscope (magnification, x40) and the invading cells were counted in each field.

Evaluation of immunohistochemistry results. The immunohistochemistry sections were scored independently by three experienced pathologists who had no prior knowledge of the patient data. To evaluate the staining results, all areas of each sample were examined and only the section with the greatest immunoreactivity was selected for quantification. For Twist2, cytoplasmic and nuclear immunoreactivity were determined. Positive cells had brown granules in the cytoplasm (15). Cytoplasmic staining was scored based on the staining intensity and percentage of positive cell nuclei. The cytoplasmic expression of Twist 2 and membrane expression of E-cadherin protein were assessed by a semi-quantitative method: the sections were assessed for the intensity of the staining (0-3) and the percentage of positively stained cells (0-3). Index of Twist 2 and E-cadherin expression was calculated as percentage $\mathrm{x}$ intensity of the staining. Therefore, score 0 presents negative (-), 1-3 serves as weak positive $(+), 4-6$ as positive $(++)$, and 7-9 as strong positive (+++) expression (15-19,21-23).

Statistical analysis. Statistical analyses were performed using SPSS 17.0 software. Clinical and histopathologic information and the results from the immunohistochemical studies of the tissue microarray were entered into a database. The Twist 2 and E-cadherin expression among different tissues was analyzed using the Kruskal-Wallis test and the clinicopathological data were analyzed with the Mann-Whitney U test. The differences between the 31 pairs of tumor and para-tumor tissues were campared using the Wilcoxon's sign rank test. For all of the statistical analyses, a two-tailed $p$-value of $\leq 0.05$ was considered statistically significant. 
Table III. Association between the clinicopathological features and the expression of E-cadherin in cervical disease tissues.

\begin{tabular}{|c|c|c|c|c|c|}
\hline & \multicolumn{2}{|c|}{ E-cadherin expression in membrane } & \multirow[b]{2}{*}{ Positive rate } & \multirow[b]{2}{*}{$\chi^{2}$} & \multirow[b]{2}{*}{ P-value } \\
\hline & - & + to +++ & & & \\
\hline Tissue type & & & & 14.156 & 0.001 \\
\hline Normal & 0 & 6 & $100.0 \%$ & & \\
\hline CIN I-II & 0 & 19 & $100.0 \%$ & & \\
\hline CIN III & 0 & 11 & $100.0 \%$ & & \\
\hline SCC & 22 & 48 & $68.6 \%$ & & \\
\hline Total $(n=106)$ & 22 & 84 & & & \\
\hline Age (years) & & & & 1.25 & 0.264 \\
\hline$\leq 45$ & 11 & 53 & $82.8 \%$ & & \\
\hline$>45$ & 11 & 31 & $73.8 \%$ & & \\
\hline Total $(n=106)$ & 22 & 84 & & & \\
\hline Differentiation of tumor cells & & & & 3.802 & 0.148 \\
\hline High & 1 & 1 & $50.0 \%$ & & \\
\hline Moderate & 10 & 33 & $76.7 \%$ & & \\
\hline Low & 11 & 14 & $56.0 \%$ & & \\
\hline Total $(n=70)$ & 22 & 48 & & & \\
\hline Lymph node metastasis & & & & 7.231 & 0.007 \\
\hline Negative & 13 & 42 & $76.4 \%$ & & \\
\hline Positive & 9 & 6 & $40.0 \%$ & & \\
\hline Total $(\mathrm{n}=70)$ & 22 & 48 & & & \\
\hline
\end{tabular}

CIN, cervical intraepithelial neoplasia; SCC, squamous cell carcinoma.

\section{Results}

Correlation between Twist 2 and E-cadherin expression and the clinicopathological parameters in all grades of cervical disease. To investigate the relationship of Twist 2 and E-cadherin expression with the pathological characteristics, we performed immunohistochemical staining for Twist2 and E-cadherin on tissue microarrays, which included 6 cases of cervical squamous epithelial tissue, 23 cases of CIN I-II tissue, 12 cases of CIN III tissue, and 70 cases of cervical squamous cell carcinoma tissue. Twist 2 expression was mainly located in the cytoplasm. The rate of Twist 2 expression gradually increased from $0 \%$ in the normal cervical squamous epithelial to $57.9 \%$ in CIN I-II, $72.7 \%$ in CIN III and $95.5 \%$ in the cervical squamous cell carcinoma tissue. The differences between the four groups were statistically significant, with $\mathrm{p}$-values $<0.05$. In addition, we found no relationship between Twist 2 expression and patient age, tumor grade, or lymph node metastasis (Table II).

The rate of E-cadherin expression in cervical squamous cell carcinoma was $68.6 \%$, which was lower than that in the normal cervical squamous epithelial and CIN tissues $(\mathrm{p}<0.05)$. In contrast to Twist 2 expression, we found that the expression of E-cadherin was related to lymph node metastasis $(\mathrm{p}<0.05)$; however, there was no significant relationship with patient age or tumor grade (Table III).

Expression of Twist 2 and E-cadherin in the progression of cervical cancer. To determine whether there was a statisti- cally significant difference in Twist 2 expression between each grade of cervical disease, we compared the following groups: normal cervical squamous epithelial vs. CIN I-II; normal cervical squamous epithelial vs. CIN III; normal cervical squamous epithelial vs. squamous cell carcinoma (SCC); CIN I-II vs. CIN III; CIN I-II vs. SCC; and CIN III vs. SCC. Our results demonstrated statistically significant differences in the above groups with the exception of the following two pairings: CIN I-II and CIN III, CIN III and SCC (Tables IV and V, Fig. 1).

We also compared E-cadherin expression in each grade of cervical disease using the same comparison groups with Twist2. We found statistically significant differences in all pairs, except for the CIN I-II and CIN III pair (Tables VI and VII, Fig. 1).

The difference in Twist 2 and E-cadherin expression between cervical tumor and tumor adjacent tissues. To evaluate the potential metastasis of cervical cancer, we analyzed the expression of Twist 2 and E-cadherin in 31 pairs of cervical tumor tissues and tumor adjacent tissues. In the cervical tumor tissues, the rate of E-cadherin expression was 77.4\% (24/31), which was lower than the rate of $96.8 \%$ (for 30/31) in the tumor adjacent tissues $(\mathrm{p}<0.05)$. In addition, the rate of Twist 2 expression in tumor tissues $(96.8 \%$ 30/31) was higher than that in the tumor adjacent tissues $(51.6 \% 16 / 31),(p<0.05)$ (Tables VIII and IX, Fig. 2). In addition, we found that the difference in E-cadherin expression between the cervical tumor and tumor adjacent tissues was statistically significant $(\mathrm{Z}=-4.602, \mathrm{p}<0.05)$; the 
Table IV. Protein expression of Twist2 in various lesions of cervical squamous epithelial.

\begin{tabular}{lrrrrrrr}
\hline & \multicolumn{2}{c}{$\begin{array}{c}\text { Twist2 expression } \\
\text { in cytoplasm }\end{array}$} & & & \\
\cline { 2 - 5 } & - & + & ++ & +++ & Total & H & P-value \\
\hline Tissue group & & & & & & 31.738 & 0.000 \\
Normal & 6 & 0 & 0 & 0 & 6 & & \\
CIN I-II & 8 & 4 & 6 & 1 & 19 & & \\
CIN III & 3 & 7 & 0 & 1 & 11 & & \\
SCC & 3 & 18 & 33 & 16 & 70 & & \\
Total & 20 & 29 & 39 & 18 & 106 & & \\
\hline
\end{tabular}

CIN, cervical intraepithelial neoplasia; SCC, squamous cell carcinoma.

Table V. Difference in the expression of Twist2 between the groups of cervical tissues.

\begin{tabular}{llcc}
\hline Tissue groups compared & $\mathrm{Z}$ & P-value \\
\hline Normal & CIN I-II & 2.766 & 0.011 \\
& CIN III & 2.389 & 0.037 \\
& SCC & 3.174 & 0.002 \\
CIN I-II & CIN III & -0.652 & 0.553 \\
& SCC & 5.027 & 0.000 \\
CIN III & SCC & 1.674 & 0.094 \\
\hline
\end{tabular}

CIN, cervical intraepithelial neoplasia; SCC, squamous cell carcinoma.
Table VI. Protein expression of E-cadherin in various lesions of cervical squamous epithelial.

\begin{tabular}{|c|c|c|c|c|c|c|c|}
\hline & \multicolumn{4}{|c|}{$\begin{array}{c}\text { E-cadherin expression } \\
\text { in membrane }\end{array}$} & \multirow[b]{2}{*}{ Total } & \multirow[b]{2}{*}{$\mathrm{H}$} & \multirow[b]{2}{*}{ P-value } \\
\hline & - & + & ++ & +++ & & & \\
\hline Tissue group & & & & & & 18.776 & 0.000 \\
\hline Normal & 0 & 0 & 0 & 6 & 6 & & \\
\hline CIN I-II & 0 & 2 & 11 & 6 & 19 & & \\
\hline CIN III & 0 & 4 & 3 & 4 & 11 & & \\
\hline SCC & 22 & 16 & 16 & 16 & 70 & & \\
\hline Total & 22 & 22 & 30 & 32 & 106 & & \\
\hline
\end{tabular}

CIN, cervical intraepithelial neoplasia; SCC, squamous cell carcinoma.

Table VII. Difference in the expression of E-cadherin between the groups of cervical tissues.

\begin{tabular}{llcc}
\hline Tissue groups compared & $\mathrm{Z}$ & P-value \\
\hline Normal & CIN I-II & 2.336 & 0.036 \\
& CIN III & 2.724 & 0.015 \\
& SCC & 4.095 & 0.000 \\
CIN I-II & CIN III & 0.182 & 0.856 \\
& SCC & 4.095 & 0.000 \\
CIN III & SCC & 3.420 & 0.001 \\
\hline
\end{tabular}

CIN, cervical intraepithelial neoplasia; SCC, squamous cell carcinoma.

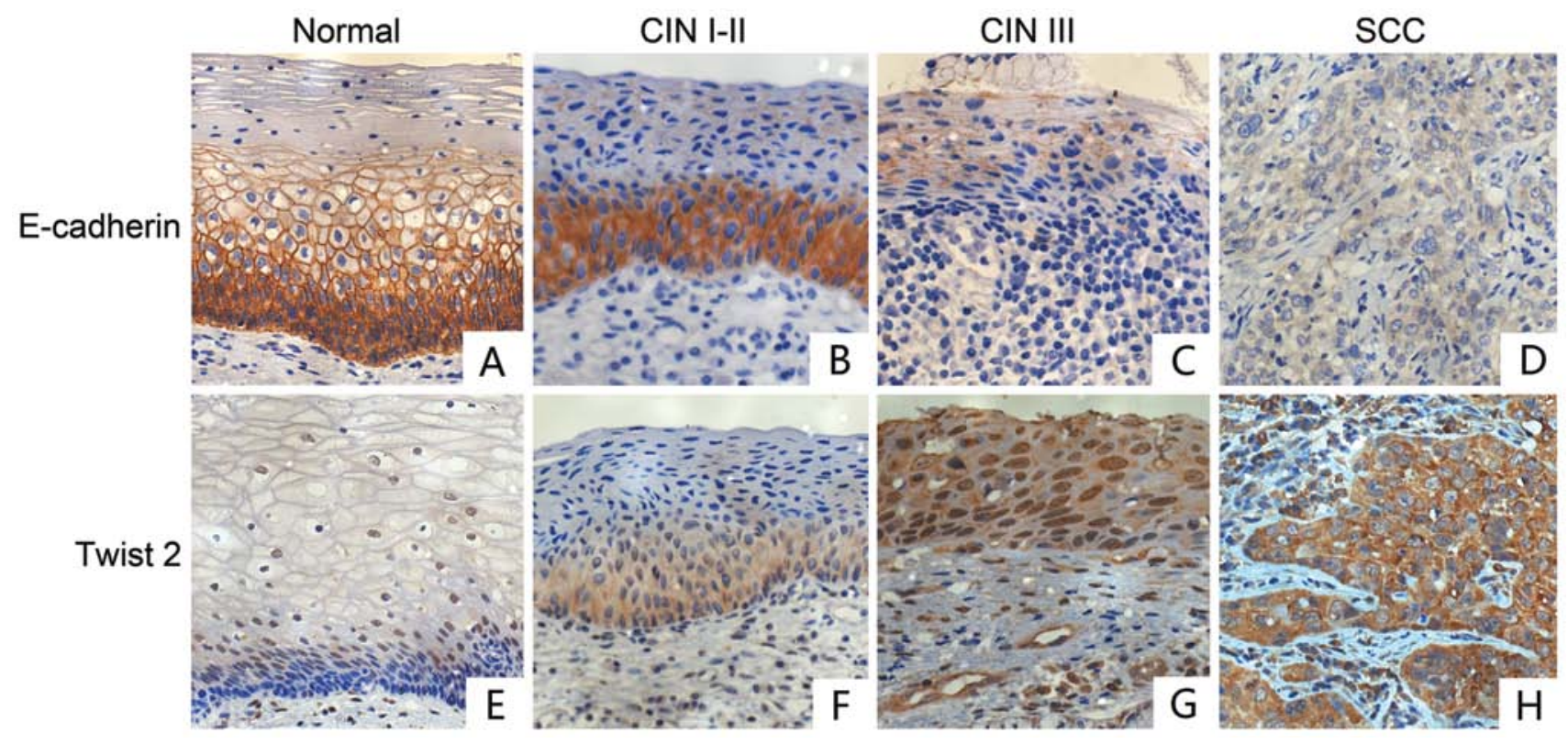

Figure 1. Immunohistochemical expression of Twist2 and E-cadherin in all grades of cervical disease (magnification, $\mathrm{x} 400$ ). (A) The expression of E-cadherin in normal cervical squamous epithelial tissue. (B) The expression of E-cadherin in cervical intraepithelial neoplasia (CIN) I-II. (C) The expression of E-cadherin in CIN III. (D) The expression of E-cadherin in cervical squamous cell carcinoma. (E) The expression of Twist2 in normal cervical squamous epithelial tissue. (F) The expression of E-cadherin in CIN I-II. (G) The expression of E-cadherin in CIN III. (H) The expression of E-cadherin in cervical squamous cell carcinoma. 
Table VIII. Expression of E-cadherin in 31 pairs of cervical tumor and tumor adjacent tissues.

\begin{tabular}{lrrrrrrr}
\hline & \multicolumn{3}{l}{ E-cadherin expression in membrane } & & \\
\cline { 2 - 5 } & - & + & ++ & +++ & Total & $\mathrm{Z}$ & P-value \\
\hline Pairs of cervical disease tissue & & & & & & -3.172 & 0.001 \\
SCC & 7 & 8 & 8 & 8 & 31 & & \\
Para-SCC & 1 & 0 & 15 & 15 & 31 & & \\
Total & 8 & 8 & 23 & 23 & 62 & & \\
\hline
\end{tabular}

CIN, cervical intraepithelial neoplasia; SCC, squamous cell carcinoma.

Table IX. Expression of Twist 2 in 31 pairs of cervical tumor and tumor adjacent tissues.

\begin{tabular}{lrrrrrrr}
\hline & \multicolumn{3}{c}{ Twist2 expression in cytoplasm } & & & \\
\cline { 2 - 5 } & \multicolumn{1}{c}{} & + & ++ & +++ & Total & $\mathrm{Z}$ & P-value \\
\hline Pairs of cervical disease tissue & 1 & 4 & 8 & 18 & 31 & -5.662 & 0.000 \\
SCC & 15 & 10 & 6 & 0 & 31 & & \\
Para-SCC & 16 & 14 & 14 & 18 & 62 & & \\
Total & & & & & & \\
\end{tabular}

CIN, cervical intraepithelial neoplasia; SCC, squamous cell carcinoma.

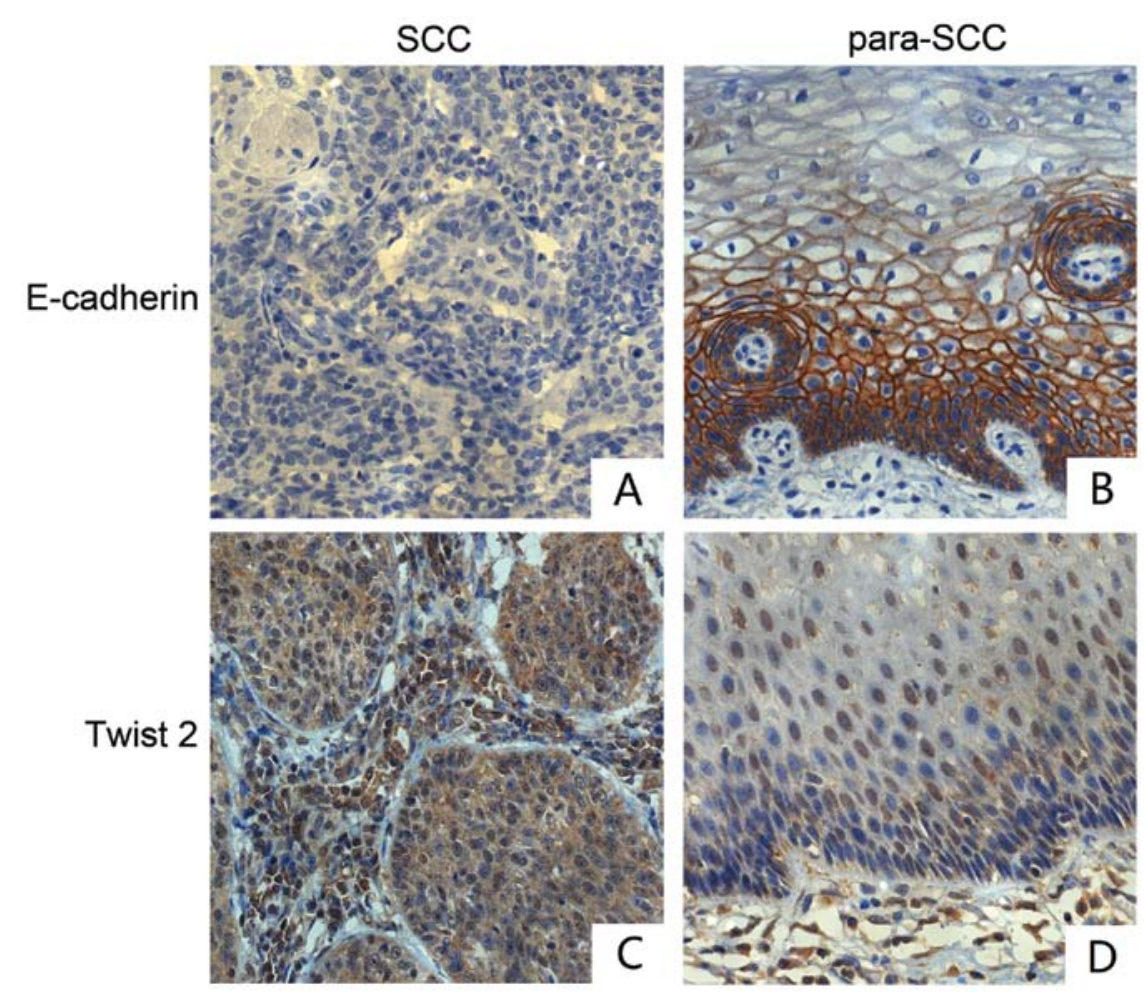

Figure 2. Expression of Twist2 and E-cadherin in 31 pairs of cervical tumor and tumor adjacent tissues (magnification, $\mathrm{x} 400$ ). (A) The expression of E-cadherin in cervical squamous cell carcinoma. (B) The expression of E-cadherin in normal squamous epithelial tissue adjacent to cervical squamous cell carcinoma. (C) The expression of Twist2 in cervical squamous cell carcinoma. (D) The expression of Twist2 in normal squamous epithelial tissue adjacent to cervical squamous cell carcinoma.

same result was found when evaluating Twist2 expression $(\mathrm{Z}=-3.383, \mathrm{p}<0.05)($ Table $\mathrm{X}$ and $\mathrm{XI})$.
HPV16 oncogenes induce EMT cell morphological alterations of SiHa cells in vitro. Above we described the clinical signifi- 
Table X. Difference in the expression of Twist2 between tumor and tumor adjacent tissue.

\begin{tabular}{lc}
\hline & Para tumor-tumor \\
\hline $\mathrm{Z}$ & $-4.602^{\mathrm{a}}$ \\
Exact Sig. (two-tailed) & 0.000 \\
\hline
\end{tabular}

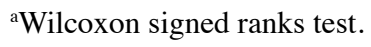
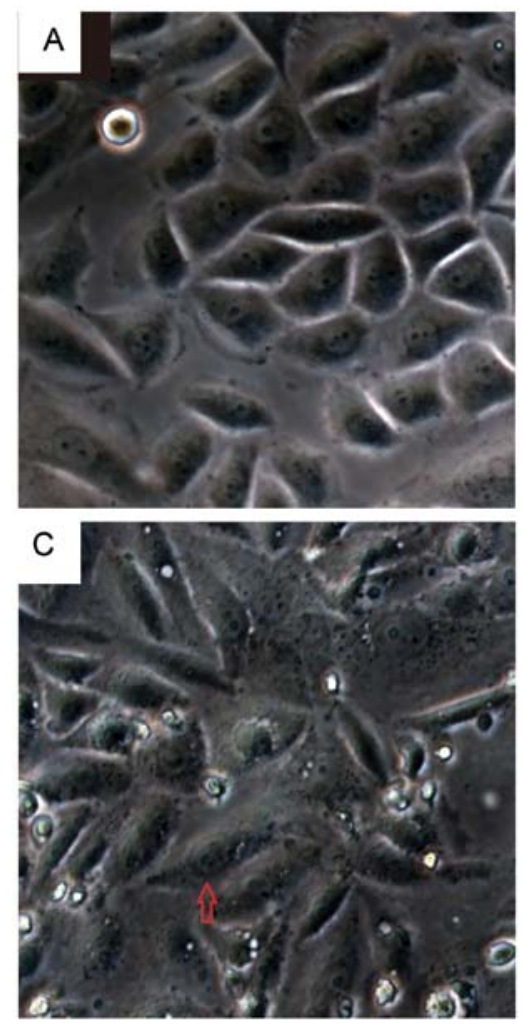

Figure 3. Morphological alterations in the SiHa cells $24 \mathrm{~h}$ after the transfection of HPV16 E2/E6/E7 (magnification, x200). (A) Control group: cells transfected with vector, (B) cells transfected with P-CAG-MYC-HPV16 E2, (C) cells transfected with P-CAG-MYC-HPV16 E6, (D) cells transfected with P-CAGMYC-HPV16 E7. The morphological alterations are indicated by the arrows.

cance of Twist2 and E-cadherin expression in the progression of cervical cancer. We next aimed to identify if HPV16 oncogenes affect the EMT process. Firstly, we transfected HPV16 E2/E6/E7 into SiHa cells in vitro, for 24 and $48 \mathrm{~h}$, respectively, to investigate whether the cellular morphology is able to be altered due to the overexpression of HPV16 oncogenes. Compared with the vector group, cells transfected with the target genes became elongated, spindle-shaped and scattered and adopted a fibroblast-like morphology. Moreover, the intracellular gap was wider than the vector control. The transition was even more obvious when cells were transfected for $48 \mathrm{~h}$ (Figs. 3 and 4).

HPV16 oncogenes upregulate the expression of Twist 2 and vimentin and downregulate E-cadherin in vitro. To validate the effect of HPV16 oncogenes on Twist 2 and vimentin, the most important genes controlling EMT in cervical cancer, we applied RT-PCR and western blotting, to detect their mRNA
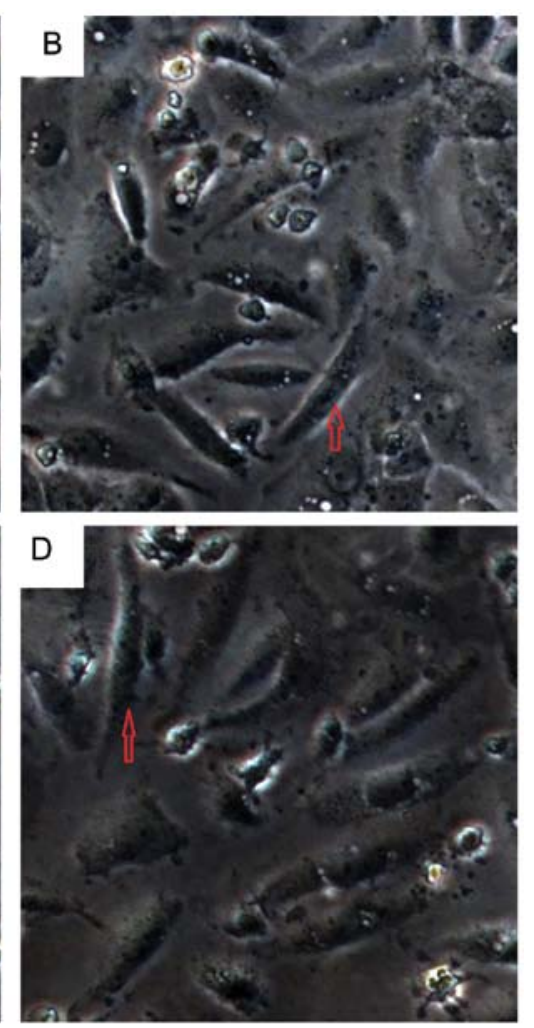

Table XI. Difference in the expression of E-cadherin between tumor and tumor adjacent tissue.

\begin{tabular}{lc}
\hline & Para tumor-tumor \\
\hline $\mathrm{Z}$ & $-3.383^{\mathrm{a}}$ \\
Exact Sig. (two-tailed) & 0.000 \\
\hline
\end{tabular}

${ }^{a}$ Wilcoxon signed ranks test 

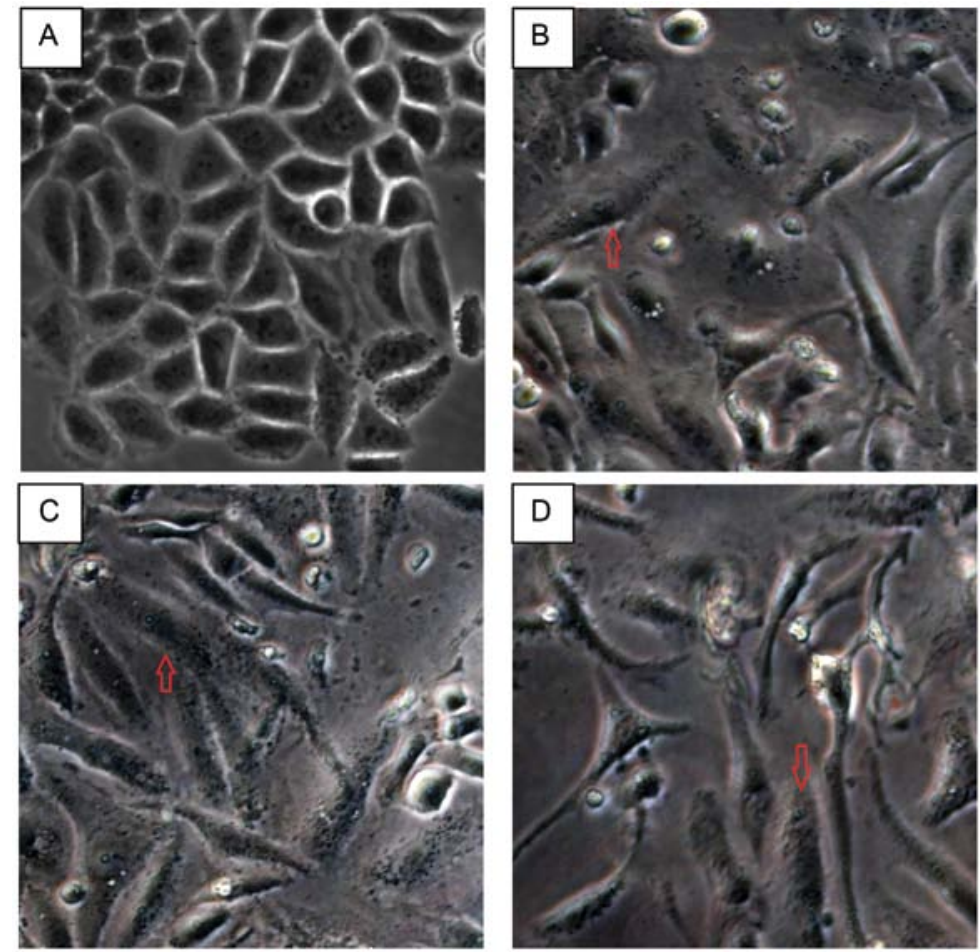

Figure 4. Morphological alterations of the SiHa cells $48 \mathrm{~h}$ after the transfection of HPV16 E2/E6/E7 (magnification, x200). (A) Control group: cells transfected with vector, (B) cells transfected with P-CAG-MYC-HPV16 E2, (C) cells transfected with P-CAG-MYC-HPV16 E6, (D) cells transfected with P-CAGMYC-HPV16 E7. The morphological alterations are indicated by the arrows.

Promoting effect of HPV16 oncogenes on the invasion of SiHa cells. We then investigated whether treatment with HPV16 oncogenes promotes the invasion of $\mathrm{SiHa}$ cell lines using Matrigel-coated Boyden chambers. First, SiHa cells were introduced with P-CAG-MYC, P-CAG-MYC-HPV16 E2, P-CAG-MYC-HPV16 E6, and P-CAG-MYC-HPV16 E7 plasmids. After $24 \mathrm{~h}$, the HPV16 E2/E6/E7-treated SiHa cells were markedly more invasive than their untreated counterparts $(\mathrm{p}<0.05)$ (Fig. 7).

\section{Discussion}

It has been proposed that tumor cells lose homotypic adhesion, change morphology, and acquire migratory capacity to become 'invasive' through the activation of an evolutionarily conserved developmental process known as epithelial-mesenchymal transition (EMT) $(24,25)$. It is well known that epithelialmesenchymal transition is closely involved in carcinogenesis, invasion, metastasis, recurrence and chemoresistance (7-14). Some molecular changes are reported to be found in the EMT process, including the following: i) decreasing expression of epithelial markers, such as E-cadherin; ii) increasing expression of mesenchymal-related proteins, such as vimentin; iii) cytoskeleton rearrangement mediated by Rho small GTPases; and iv) upregulation and nuclear translocation of transcription factors, such as Twist2, Snail and Zeb (14).

The transcription factors Twist 2 and Twist 1 are vital members of the basic helix-loop-helix (bHLH) family. Both of these molecules are able to bind to the E-boxes of E-cadherin to repress the expression of the CDH1 (E-cadherin) gene and induce the EMT process $(13,14,23)$. Recently, a great deal of effort has been made in investigating whether Twist 2 is an effective biomarker with which to predict and evaluate cancer progression. Gasparotto et al (26) confirmed that overexpression of Twist 2 is intimately related to the poor prognosis of patients with head and neck squamous cell carcinoma. Li et al (15) found that upregulation of Twist2, in combination with aberrant E-cadherin expression in primary cervical cancer tissues, may indicate malignant transformation and distant metastasis.

It has been suggested that the Twist 2 protein is located in both the cytoplasm and nucleus $(15,27)$. In the present study, we did not evaluate the 7 cases in which Twist 2 was located mainly in the nucleus, which mostly was noted in the normal cervical squamous epithelial and CIN I-II tissues. We primarily focused on cytoplasmic staining. This analysis demonstrated that Twist 2 staining was gradually increased from $0 \%$ in normal cervical squamous epithelial to $57.9 \%$ in CIN I-II (mild to moderate atypical hyperplasia ), $72.7 \%$ in CIN III (severe atypical hyperplasia or carcinoma in situ) and 95.5\% in cervical squamous cell carcinoma. This intriguing finding indicates that Twist 2 upregulation is a possible predictive indicator of the malignant potential for cervical disease. Moreover, the expression of Twist 2 could also partly be valuable for evaluating the grades of cervical lesions, although there was no statistical significance between CIN I-II and CIN III ( $p>0.05)$, or CIN III and SCC (CIN III only 11 cases) ( $p>0.05)$.

It is known that a reduction in the epithelial adhesion protein $\mathrm{E}$-cadherin and an increase in $\mathrm{N}$-cadherin and the mesenchymal intermediate filament protein vimentin mark the EMT process (17). A similar pattern was observed in a study performed by Myong et al (28). In this study it was proven that E-cadherin and vimentin were effective indicators of EMT in evaluating cervical cancer progression by 
A
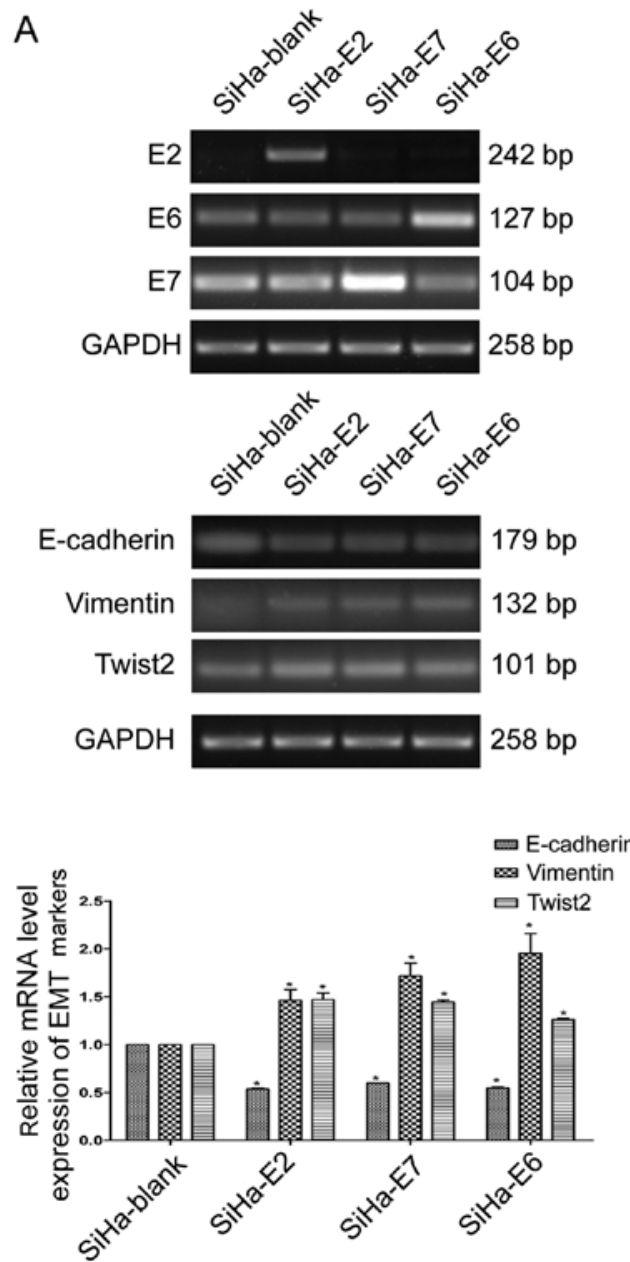
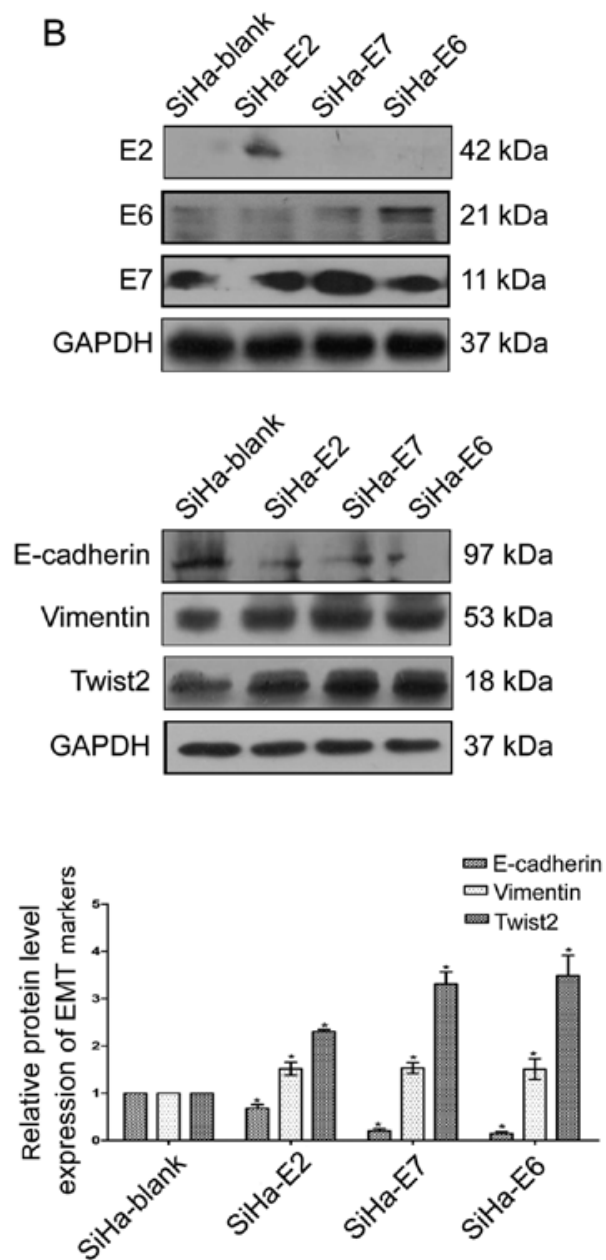

Figure 5. Expression of epithelial-mesenchymal transition (EMT) markers in SiHa cells: (A and B) The p-CAG-MYC-HPV16 E2/E6/E7 plasmids were successfully transfected into SiHa cells both at the mRNA and protein levels. HPV16 E2/E6/E7 influenced the expression of EMT indicators: upregulating the expression of Twist 2 and vimentin while downregulating the expression of E-cadherin both at the mRNA and protein levels. "P<0.05, compared with the SiHa-blank group.
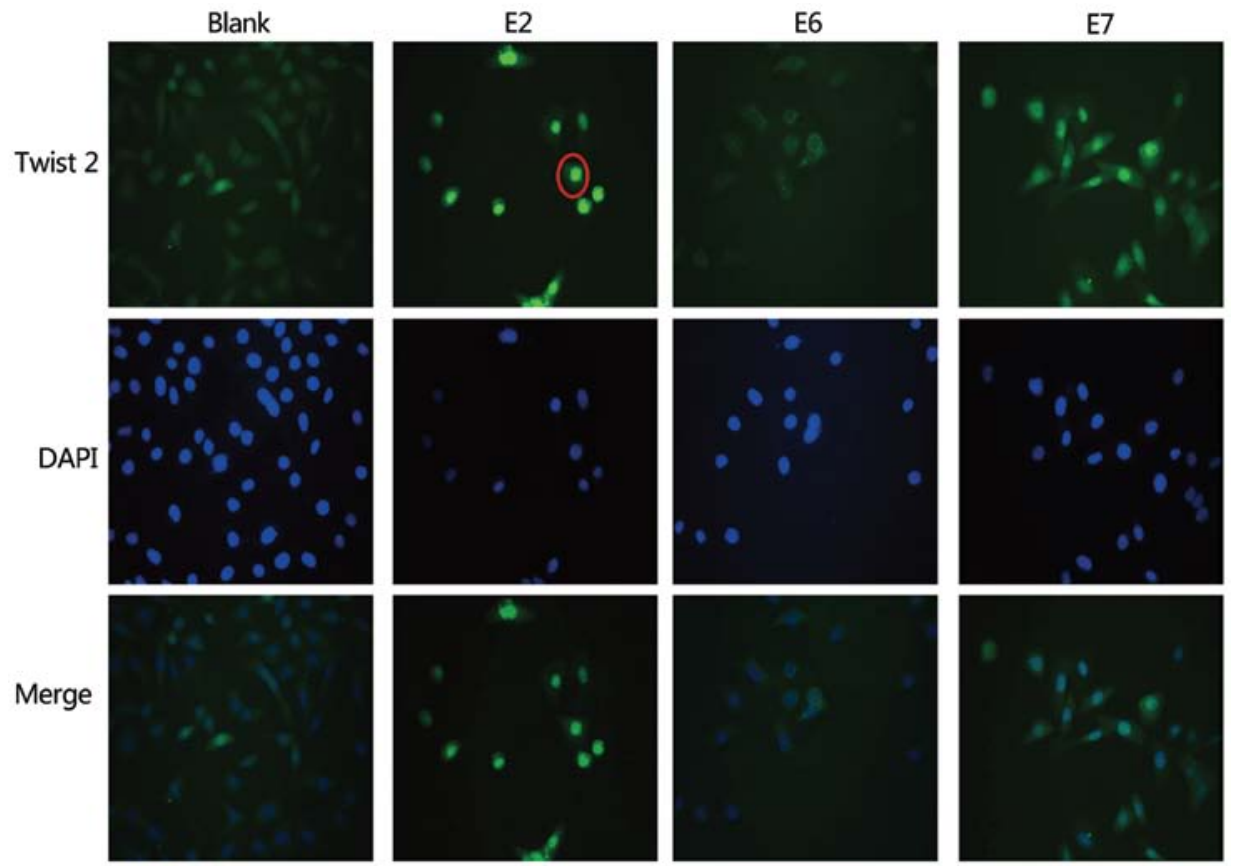

Figure 6. Localization of Twist2 in the SiHa cell line. Immunofluorescence micrographs show the location of Twist2 in the SiHa cells, HPV E2 plasmidintroduced SiHa cells, HPV E6 plasmid-introduced SiHa cells and HPV E7 plasmid-introduced SiHa cells, respectively. Ovals mark the zones of Twist2 expression, which indicated a significantly higher intensity in the nucleus compared with the cytoplasm. Original magnification, $\mathrm{x} 400$. 

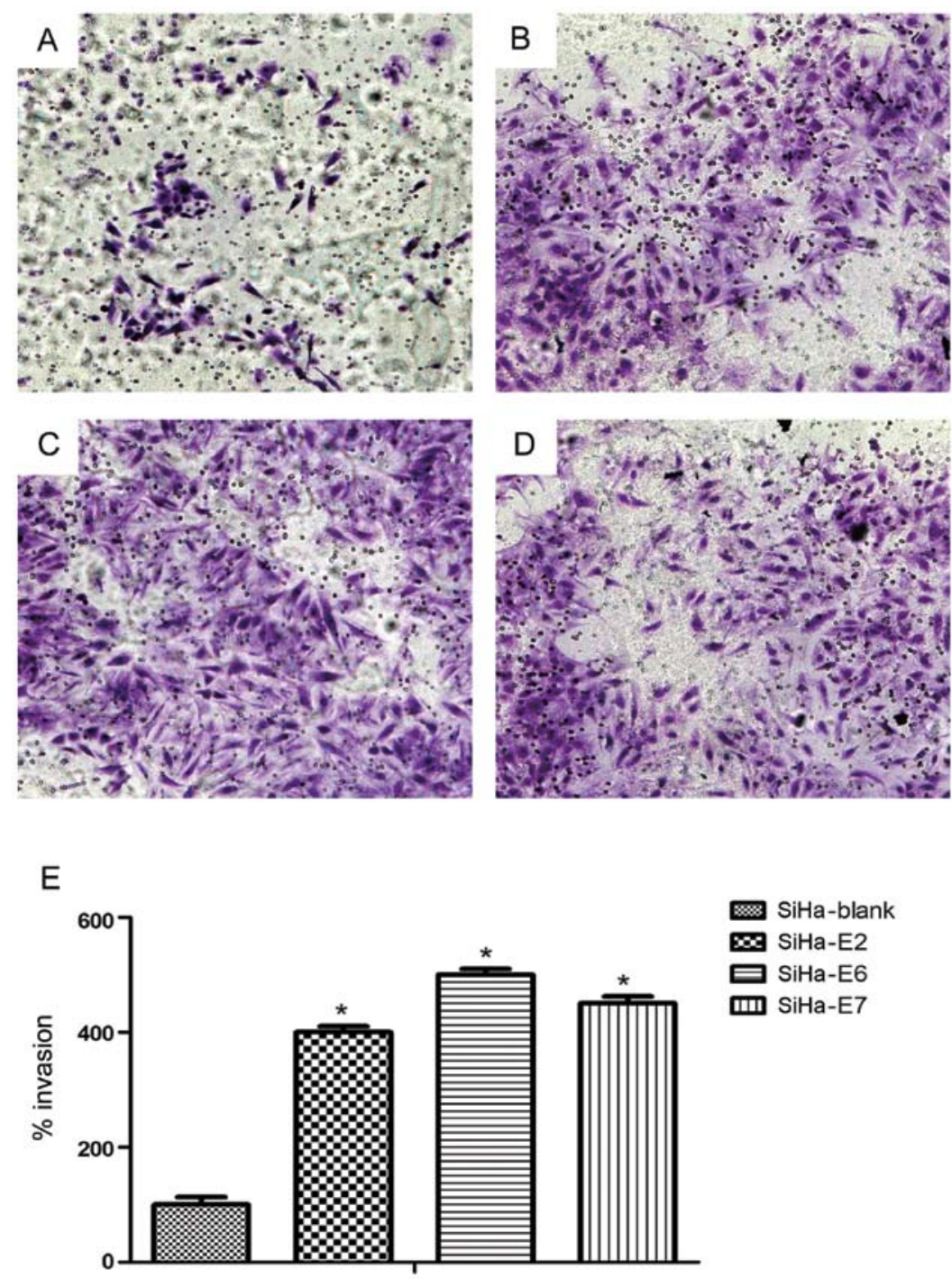

Figure 7. Effect of E2, E6, E7 on the invasive capability of SiHa cells. Microphotographs show representative fields from the Giemsa-stained lower membranes of the Boyden chambers: (A) SiHa-blank, (B) SiHa-E2, (C) SiHa-E6 and (D) SiHa-E7 cell groups. (E) Histogram shows the number of untreated and E2, E6, E7 plasmids-treated cells (expressed as a percentage of the untreated cells assigned as 100). " $\mathrm{P}<0.05$, compared with the SiHa-blank group.

immunohistochemical staining. In the present study, although the expression of E-cadherin could not be differentiated between CIN I-II and CIN III ( $p>0.05)$, it is still quite obvious that the expression of E-cadherin in cervical cancer tissues was lower than that in normal cervical squamous epithelial and cervical squamous cell carcinoma tissues. Cancer cells can convert to the epithelial state through the process of mesenchymal to epithelial transition (MET), which is highly indicative of the reversibility of EMT (29). This reversible process allows gene expression to be rapidly switched according to developmental and micro-environmental signals. A hybrid cell which contains both epithelial and mesenchymal traits can be generated by incomplete EMT into an epithelial cell (30). This may account for the reason why there was no significant difference in Twist 2 expression between the CIN I-II and CIN III, or CIN III and SCC tissues and why there was no significant difference in E-cadherin expression between the CIN I-II and CIN III tissues in the immunohistochemical staining. The limited number of cases of immunohistochemistry samples may be another reason.
Lee et al (31) and Hsu et al (32) demonstrated that EMT is intimately related to the invasion of cervical cancer and particularly the progression of malignant tumors through clinical tissue observations. It is believed that EMT plays a vital role in the intravasation of cells into the vascellum as well as the lymph vessels, thus shaping micrometastases as the most essential process during EMT is the degradation of the basal membrane (29). Needless to say, it is widely believed that EMT plays an indispensable role during the process of cancer malignant metastasis $(7-14,23,26-28)$. In the present study, we found that E-cadherin expression was lower in the tumor tissues than that in the tumor adjacent tissues $(p<0.05)$, and Twist 2 expression was higher in tumor tissues than that in the tumor adjacent tissues $(\mathrm{p}<0.05)$ (Tables VIII and IX, Fig. 2). In addition, the difference in the expression of Twist 2 between the tumor tissues and tumor adjacent tissues was statistically significant $(Z=-4.602, p<0.05)$; the same was true for the difference in $\mathrm{E}-\mathrm{cadherin}$ expression $(\mathrm{Z}=-3.383, \mathrm{p}<0.05)$. Based on the multiple anti-proliferative functions, anti-invasion and anti-metastasis properties of E-cadherin, the loss of E-cadherin 
tends to enhance the metastatic diffusion in a large number of cancer types (33). There are several mechanisms involved in the loss of E-cadherin, such as genetic mutation, epigenetic silencing and transcription repression (14). By evaluating the differences in the expression of Twist 2 and E-cadherin at the same time between 31 pairs of cervical tumor and tumor adjacent tissues, we were able to demonstrate that joint detection of the expression of Twist 2 and E-cadherin could better predict cervical carcinogenesis and progression.

Previous studies have suggested that high-risk HPV oncoproteins may contribute to EMT. Recently, studies have reported that HPV16 oncogenes are correlated with the EMT process during cervical progression and metastasis. Jung et al (34) found that HPV16 induces EMT-like processes via induction of EMT transcription factors which may contribute to tumor progression and metastasis. HPV18 E6 expression was found to be correlated with a fibroblastoid morphology in SV40immortalized human keratinocytes (35). A microarray analysis suggested that the modulation of a great number of genes are involved in keratinocyte differentiation and EMT mediated by HPV16 E6 (36). In addition, Hellner et al (17) reported that the oncogenic HPV16 E7 protein in normal human epithelial cells leads to increased levels of vimentin as well as reduced levels of the epithelial adhesion protein E-cadherin. In the present study, HPV16 E6/E7 induced the morphological conversion of SiHa cells from a cobblestone-shaped epithelium-like phenotype to a spindle-shaped mesenchyme-like phenotype. Corresponding to the morphological alterations, HPV16 E6/E7 also influenced the expression of EMT-relative indicators, such as upregulation of Twist 2 and vimentin and downregulation of E-cadherin. It is worth noting that these results were also manifested in the SiHa cells transfected with the HPV16 E2 plasmid. The HPV16 E2 protein is a multifunctional DNA-binding transcription factor that plays a pivotal role in transcriptional regulation and viral DNA replication. In addition, this protein modulates host cells through direct protein interactions (37-41) and is therefore potentially responsible for HPV-induced carcinogenesis (42). According to our results, HPV16 E2 may induce Twist 2 translocation from the cytoplasm to the nucleus to fully exert its function as a transcription factor (Fig. 6). Since Twist2 serves as a transcription factor which binds to the E-boxes of E-cadherin to repress its expression and induce the EMT process $(13,14,23)$, our findings indicate that the HPV oncogene E2 can actually upregulate the expression of Twist 2 and downregulate E-cadherin. We then speculated that Twist2 may pass through the nuclear envelope to interact with HPV E2 and continue to extend the function of E-cadherin suppression and EMT induction. While the mechanism of E-cadherin downregulation in HPV16 E2/E6/E7-transfected SiHa cells remains to be explored, our results are similar to a recent investigation that demonstrated that E-cadherin expression was preserved when HPV16 E7 was silenced by siRNA in HPV16-transformed human keratinocytes, irrespective of the transcription factors slug and snail (19). Moreover, in the present study, we found that HPV16 oncogenes could promote the invasive ability of SiHa cells in vitro. This once again identified a new metastatic potential of HPV16 oncogenes in cervical carcinogenesis. Thus, we believe that HPV16 oncogenes can actually induce EMT-like processes which may contribute to cancer carci- nogenesis and progression. However, the specific mechanism remains to be elucidated.

In conclusion, we demonstrated that joint detection of Twist 2 and E-cadherin expression can be used to predict and evaluate carcinogenesis and progression of cervical cancer. HPV16 oncogenes can induce EMT-like processes in SiHa cells and contribute to the EMT process via regulating the expression of Twist 2, E-cadherin and vimentin. These results provide new insight into the mechanism of cervical carcinogenesis and progression. In addition, joint detection of E-cadherin and Twist 2 expression could aid in distinguishing and diagnosing early stage cervical cancer to improve the survival rate of cervical cancer patients.

\section{Acknowledgements}

This study was supported by grants from the National Natural Science Foundation of China (81201541), the Shanghai Pujiang Program (12PJD002), and the Shanghai Science and Technology Committee Foundation (09411962500).

\section{References}

1. zur Hausen H: Papillomaviruses and cancer: from basic studies to clinical application. Nat Rev Cancer 2: 342-350, 2002.

2. Faridi R, Zahra A, Khan K and Idrees M: Oncogenic potential of human papillomavirus (HPV) and its relation with cervical cancer. Virol J 8: 269, 2011.

3. Panjković $M$ and Ivković-Kapicl T: Etiology and pathogenesis of precancerous lesions and invasive cervical carcinoma. Med Pregl 61: 364-368, 2008 (In Serbian).

4. Snijders PJ, Steenbergen RD, Heideman DA and Meijer CJ: HPV-mediated cervical carcinogenesis: concepts and clinical implications. J Pathol 208: 152-164, 2006.

5. Javier MG and Sastre-Garau X: Uterine cervix carcinoma: recent biological data and update for improving follow-up and treatment. Isr Med Assoc J 14: 700-704, 2012.

6. Ghittoni R, Accardi R, Hasan U, Gheit T, Sylla B and Tommasino M: The biological properties of E6 and E7 oncoproteins from human papillomaviruses. Virus Genes 40: 1-13, 2010.

7. De Craene B and Berx G: Regulatory networks defining EMT during cancer initiation and progression. Nat Rev Cancer 13: 97-110, 2013.

8. Stewart CJ and McCluggage WG: Epithelial-mesenchymal transition in carcinomas of the female genital tract. Histopathology 62: 31-43, 2013.

9. Lim J and Thiery JP: Epithelial-mesenchymal transitions: insights from development. Development 139: 3471-3486, 2012.

10. Le Bras GF, Taubenslag KJ and Andl CD: The regulation of cell-cell adhesion during epithelial-mesenchymal transition, motility and tumor progression. Cell Adh Migr 6: 365-373, 2012.

11. Rodriguez FJ, Lewis-Tuffin LJ and Anastasiadis PZ: E-cadherin's dark side: possible role in tumor progression. Biochim Biophys Acta 1826: 23-31, 2012.

12. Iwatsuki M,Mimori K, Yokobori T, et al: Epithelial-mesenchymal transition in cancer development and its clinical significance. Cancer Sci 101: 293-299, 2010.

13. Sanchez-Tillo E, Liu Y, de Barrios O, et al: EMT-activating transcription factors in cancer: beyond EMT and tumor invasiveness. Cell Mol Life Sci 69: 3429-3456, 2012.

14. Lee MY and Shen MR: Epithelial-mesenchymal transition in cervical carcinoma. Am J Transl Res 4: 1-13, 2012.

15. Li Y, Wang W, Wang W, et al: Correlation of TWIST2 up-regulation and epithelial-mesenchymal transition during tumorigenesis and progression of cervical carcinoma. Gynecol Oncol 124: 112-118, 2012.

16. Chamulitrat W, Schmidt R, Chunglok W, Kohl A and Tomakidi P: Epithelium and fibroblast-like phenotypes derived from HPV16 E6/E7-immortalized human gingival keratinocytes following chronic ethanol treatment. Eur J Cell Biol 82: 313-322, 2003. 
17. Hellner K, Mar J, Fang F, Quackenbush J and Munger K: HPV16 E7 oncogene expression in normal human epithelial cells causes molecular changes indicative of an epithelial to mesenchymal transition. Virology 391: 57-63, 2009.

18. Cheng YM, Chou CY, Hsu YC, Chen MJ and Wing LY: The role of human papillomavirus type $16 \mathrm{E} 6 / \mathrm{E} 7$ oncoproteins in cervical epithelial-mesenchymal transition and carcinogenesis. Oncol Lett 3: 667-671, 2012.

19. Caberg JH, Hubert PM, Begon DY, et al: Silencing of E7 oncogene restores functional E-cadherin expression in human papillomavirus 16-transformed keratinocytes. Carcinogenesis 29: 1441-1447, 2008.

20. Mühlen S, Behren A, Iftner T and Simon C: Influence of HPV16 E2 and its localisation on the expression of matrix metalloproteinase-9. Int J Oncol 37: 337-345, 2010.

21. Zhang J, Yang Y, Zhang Z, et al: Gankyrin plays an essential role in estrogen-driven and GPR30-mediated endometrial carcinoma cell proliferation via the PTEN/PI3K/AKT signaling pathway. Cancer Lett 339: 279-287, 2013.

22. Wang YX, Zhang XY, Zhang BF, Yang CQ and Gao HJ: Study on the clinical significance of Argonaute 2 expression in colonic carcinoma by tissue microarray. Int J Clin Exp Pathol 6: 476-484 2013.

23. Franco HL, Casasnovas J, Rodriguez-Medina JR and Cadilla CL: Redundant or separate entities? - roles of Twist1 and Twist 2 as molecular switches during gene transcription. Nucleic Acids Res 39: 1177-1186, 2011.

24. Zeisberg M and Neilson EG: Biomarkers for epithelial-mesenchymal transitions. J Clin Invest 119: 1429-1437, 2009.

25. Thiery JP, Acloque H, Huang RY and Nieto MA: Epithelialmesenchymal transitions in development and disease. Cell 139: 871-890, 2009

26. Gasparotto D, Polesel J, Marzotto A, et al: Overexpression of TWIST2 correlates with poor prognosis in head and neck squamous cell carcinomas. Oncotarget 2: 1165-1175, 2011.

27. Mao Y, Zhang N, Xu J, Ding Z, Zong R and Liu Z: Significance of heterogeneous Twist 2 expression in human breast cancers. PloS One 7: e48178, 2012.

28. Myong NH: Loss of E-cadherin and acquisition of vimentin in epithelial-mesenchymal transition are noble indicators of uterine cervix cancer progression. Korean J Pathol 46: 341-348, 2012.

29. Thiery JP and Sleeman JP: Complex networks orchestrate epithelial-mesenchymal transitions. Nat Rev Mol Cell Biol 7: 131-142, 2006.
30. Reik W: Stability and flexibility of epigenetic gene regulation in mammalian development. Nature 447: 425-432, 2007.

31. Lee MY, Chou CY, Tang MJ and Shen MR: Epithelialmesenchymal transition in cervical cancer: correlation with tumor progression, epidermal growth factor receptor overexpression, and snail up-regulation. Clin Cancer Res 14: 4743-4750, 2008.

32. Hsu YM, Chen YF, Chou CY, et al: $\mathrm{KCl}$ cotransporter-3 down-regulates E-cadherin/beta-catenin complex to promote epithelial-mesenchymal transition. Cancer Res 67: 11064-11073, 2007.

33. van Roy F and Berx G: The cell-cell adhesion molecule E-cadherin. Cell Mol Life Sci 65: 3756-3788, 2008.

34. Jung YS, Kato I and Kim HR: A novel function of HPV16-E6/E7 in epithelial-mesenchymal transition. Biochem Biophys Res Commun 435: 339-344, 2013.

35. Watson RA, Thomas M, Banks L and Roberts S: Activity of the human papillomavirus E6 PDZ-binding motif correlates with an enhanced morphological transformation of immortalized human keratinocytes. J Cell Sci 116: 4925-4934, 2003.

36. Duffy CL, Phillips SL and Klingelhutz AJ: Microarray analysis identifies differentiation-associated genes regulated by human papillomavirus type 16 E6. Virology 314: 196-205, 2003.

37. Thierry F: Transcriptional regulation of the papillomavirus oncogenes by cellular and viral transcription factors in cervical carcinoma. Virology 384: 375-379, 2009.

38. Forma E, Wójcik-Krowiranda K, Bieńkiewicz A and Bryś M: Molecular basis of gynecological oncology - TopBP1 protein and its participation in the transcription process. Ginekol Pol 83: 363-367, 2012 (In Polish)

39. Bodily JM, Hennigan C, Wrobel GA and Rodriguez CM: Regulation of the human papillomavirus type 16 late promoter by E7 and the cell cycle. Virology 443: 11-19, 2013.

40. Xue Y, Lim D, Zhi L, He P, Abastado JP and Thierry F: Loss of HPV16 E2 protein expression without disruption of the E2 ORF correlates with carcinogenic progression. Open Virol J 6: 163-172, 2012

41. Ramirez-Salazar E, Centeno F, Nieto K, Valencia-Hernandez A, Salcedo M and Garrido E: HPV16 E2 could act as down-regulator in cellular genes implicated in apoptosis, proliferation and cell differentiation. Virol J 8: 247, 2011.

42. Bellanger S, Tan CL, Xue YZ, Teissier S and Thierry F: Tumor suppressor or oncogene? A critical role of the human papillomavirus (HPV) E2 protein in cervical cancer progression. Am J Cancer Res 1: 373-389, 2011. 